\title{
CENTRE-BY-METABELIAN LIE ALGEBRAS
}

\author{
M. R. VAUGHAN-LEE
}

(Received 11 September 1970)

Communicated by G. E. Wall

If $V$ is a variety of metabelian Lie algebras then $V$ has a finite basis for its laws [3]. The proof of this result is similar to Cohen's proof that varieties of metabelian groups have the finite basis property [1]. However there are centreby-metabelian Lie algebras of characteristic 2 which do not have a finite basis for their laws [4]; this contrasts with McKay's recent result that varieties of centre-by-metabelian groups do have the finite basis property [2]. The rollowing theorem shows that once again " 2 " is the odd man out.

THEOREM. If $V$ is a variety of centre-by-metabelian Lie algebras over a field $K$, and if the characteristic of $K$ is not 2, then $V$ has a finite basis for its laws.

The notation will follow [4]. Throughout this paper $K$ will denote a field whose characteristic is not 2.

Let $X$ be the free Lie algebra over $K$ freely generated by $x_{1}, x_{2}, \cdots$. Then the variety of centre-by-metabelian Lie algebras over $K$ is determined by the law $\left(\left(x_{1} x_{2}\right)\left(x_{3} x_{4}\right)\right) x_{5}$. Let $F=X /\left(X^{2}\right)^{2} X$ and for $i=1,2, \cdots$ let $y_{i}$ denote the image of $x_{i}$ under the canonical epimorphism from $X$ onto $F$. Then $F$ is the free centreby-metabelian Lie algebra over $K$ freely generated by $y_{1}, y_{2}, \cdots$.

The theorem is equivalent to the following proposition.

Proposition. F satisfies the ascending chain condition on fully invariant ideals.

Now if $V$ is a variety of metabelian Lie algebras then $V$ has a finite basis for its laws [3], and so $F /\left(F^{2}\right)^{2}$ satisfies the ascending chain condition on fully invariant ideals. It follows that to prove the proposition it is sufficient to show that $F$ satisfies the ascending chain condition on fully invariant ideals of $F$ contained in $\left(F^{2}\right)^{2}$. The proof follows the method developed by Cohen in [1].

For each element $g \in\left(F^{2}\right)^{2}$ I shall define the weight of $g$, an element $w t$ $g \in\left(F^{2}\right)^{2}$. I shall define a partial well ordering, $\preccurlyeq$, and a well ordering, $\leqq$, on the set $S$ of weights of elements of $\left(F^{2}\right)^{2}$. (A partially ordered set $(S, \preccurlyeq)$ is said to be 
partially well ordered if every infinite sequence of elements of $S$ contains an ascending subsequence. This is equivalent to the property that for every subset $T \subseteq S$ there is a finite subset $T_{0} \subseteq T$ such that for each $t \in T$ there is an element $s \in T_{0}, s \preccurlyeq t$.) The partial well ordering $\preccurlyeq$ and the well ordering $\leqq$ will be used to show that fully invariant ideals of $F$ contained in $\left(F^{2}\right)^{2}$ are finitely generated as fully invariant ideals. This is equivalent to the ascending chain condition on fully invariant ideals of $F$ contained in $\left(F^{2}\right)^{2}$.

All products will be left-normed; thus $a b c$ denotes $(a b) c$.

If $a, b$ are elements of a Lie algebra then let $a b^{0}=a,\left(a b^{i-1}\right) b$ for $i=1,2, \cdots$.

Let $\Phi$ be the set of one-one order preserving maps of the positive integers into the positive integers.

Let $A$ be the set of infinite sequences of finite support of non-negative integers. Addition of elements of $A$ is defined componentwise, i.e.

$$
\left(\alpha_{1}, \alpha_{2}, \cdots\right)+\left(\beta_{1}, \beta_{2}, \cdots\right)=\left(\alpha_{1}+\alpha_{2}, \beta_{1}+\beta_{2}, \cdots\right) .
$$

Define a partial ordering $\leqq$ on $A$ by

$$
\left(\alpha_{1}, \alpha_{2}, \cdots\right) \leqq\left(\beta_{1}, \beta_{2}, \cdots\right)
$$

if $\alpha_{i} \leqq \beta_{i}$ for $i=1,2, \cdots$. If $\phi \in \Phi$ and $\alpha=\left(\alpha_{1}, \alpha_{2}, \cdots\right) \in A$ let

$$
\alpha \phi=\left(\beta_{1}, \beta_{2}, \cdots\right)
$$

where $\beta_{i}=0$ if $i \notin \operatorname{Im} \phi, \beta_{i \phi}=\alpha_{i}$ for $i=1,2, \cdots$.

If $i, j, k, l$ are positive integers, and if $\alpha=\left(\alpha_{1}, \alpha_{2}, \cdots, \alpha_{m}, 0,0, \cdots\right) \in A$ let

$$
(i, j, k, l ; \alpha)
$$

denote the element

$$
\left(y_{i} y_{j} y_{1}^{\alpha_{1}} y_{2}^{\alpha_{2}} \cdots y_{m}^{\alpha_{m}}\right)\left(y_{k} y_{l}\right)
$$

of $\left(F^{2}\right)^{2}$. By 2.8 of [4] the set

$$
S=\{(i, j, k, l ; \alpha): i, j, k, l \text { positive integers, } \alpha \in A\}
$$

spans $\left(F^{2}\right)^{2}$ as a vector space over $K$.

Define a partial ordering $\preccurlyeq$ on $S$ as follows. Let

$$
(i, j, k, l ; \boldsymbol{\alpha}) \preccurlyeq(p, q, r, s ; \boldsymbol{\beta})
$$

if there is an element $\phi \in \Phi$ such that

(1) $i \phi=p, j \phi=q, k \phi=r, l \phi=s$,

(2) $\alpha \phi \leqq \beta$,

and if

(3) $\sum_{n=1}^{\infty} \alpha_{n} \equiv \sum_{n=1}^{\infty} \beta_{n} \bmod 2$, where $\alpha=\left(\alpha_{1}, \alpha_{2}, \cdots\right), \boldsymbol{\beta}=\left(\beta_{1}, \beta_{2}, \cdots\right)$. 
Let $\preccurlyeq *$ denote the partial ordering of $S$ determined by properties (1) and (2). Then by Proposition $4.4[5](S, \preccurlyeq *)$ is partially well ordered. Hence every infinite sequence of elements of $S$ contains a subsequence which is ascending with respect to $\preccurlyeq *$. This subsequence must contain a subsequence which also satisfies property (3). Hence $(S, \preccurlyeq)$ is partially well ordered.

Defined a full ordering $\leqq$ on $S$ as follows.

Let

$$
\left(i, j, k, l ;\left(\alpha_{1}, \alpha_{2}, \cdots\right)\right)<\left(p, q, r, s ;\left(\beta_{1}, \beta_{2}, \cdots\right)\right)
$$

if one of the following conditions holds

(a) $i<p$.

(b) $i=p, j<q$.

(c) $i=p, j=q, k<r$.

(d) $i=p, j=q, k=r, l<s$.

(e) $i=p, j=q, k=r, l=s$, and, for some $n, \alpha_{n}<\beta_{n}, \alpha_{m}=\beta_{m}$ for $m>n$.

Then $(S, \leqq)$ is well ordered.

Let $g \in\left(F^{2}\right)^{2}, g \neq 0$. Then $g$ can be written as a linear combination

$$
\lambda_{1} s_{1}+\lambda_{2} s_{2}+\cdots+\lambda_{n} s_{n}
$$

where $\lambda_{1}, \lambda_{2}, \cdots, \lambda_{n}$ are non-zero elements of $K$ and $s_{1}, s_{2}, \cdots, s_{n}$ are distinct elements of $S$. Let the weight of $g$, wt $g$, be the greatest element under $\leqq$ of the set $\left\{s_{1}, s_{2}, \cdots, s_{n}\right\}$. (Strictly speaking I have defined the weight of the particular epresentation $\lambda_{1} s_{1}+\lambda_{2} s_{2}+\cdots+\lambda_{n} s_{n}$ of $g$.)

LemMA 1. If $\boldsymbol{\beta}=\left(\beta_{1}, \beta_{2}, \cdots\right) \in A$ and $\sum_{n=1}^{\infty} \beta_{n} \equiv 0 \bmod 2$ and if $(i, j, k, l ; \alpha)$ $\in S$ then the fully invariant ideal of $F$ generated by $(i, j, k, l ; \alpha)$ contains $(i, j, k, l ; \boldsymbol{\alpha}+\boldsymbol{\beta})$.

Proof. Let $\theta$ be the endomorphism of $F$ determined by

for $r=1,2, \cdots$. Then

$$
y_{r} \theta=y_{r}+y_{r} y_{n}
$$

$$
\begin{aligned}
\left(y_{1} y_{2}\right) \theta & =\left(y_{1}+y_{1} y_{n}\right)\left(y_{2}+y_{2} y_{n}\right) \\
& =y_{1} y_{2}+\left(y_{1} y_{n}\right) y_{2}+y_{1}\left(y_{2} y_{n}\right)+\left(y_{1} y_{n}\right)\left(y_{2} y_{n}\right) \\
& =y_{1} y_{2}+y_{1} y_{2} y_{n}+\left(y_{1} y_{n}\right)\left(y_{2} y_{n}\right) \text { by the Jacobi identity } \\
& =y_{1} y_{2}+y_{1} y_{2} y_{n} \text { modulo }\left(F^{2}\right)^{2} .
\end{aligned}
$$

By induction

$$
\left(y_{1} y_{2} \cdots y_{m}\right) \theta=y_{1} y_{2} \cdots y_{m}+y_{1} y_{2} \cdots y_{m} y_{n} \text { modulo }\left(F^{2}\right)^{2} .
$$

Hence 


$$
\begin{aligned}
\left(\left(y_{1} y_{2} \cdots y_{m}\right)\left(y_{m+1} y_{m+2}\right)\right) \theta & \quad \text { where } g, h \in\left(F^{2}\right)^{2} \\
= & \left(y_{1} y_{2} \cdots y_{m}+y_{1} y_{2} \cdots y_{m} y_{n}+g\right)\left(y_{m+1} y_{m+2}+y_{m+1} y_{m+2} y_{n}+h\right) \\
= & \left(y_{1} y_{2} \cdots y_{m}\right)\left(y_{m+1} y_{m+2}\right) \\
& +\left(y_{1} y_{2} \cdots y_{m} y_{n}\right)\left(y_{m+1} y_{m+2}\right)+\left(y_{1} y_{2} \cdots y_{m}\right)\left(y_{m+1} y_{m+2} y_{n}\right) \\
& +\left(y_{1} y_{2} \cdots y_{m} y_{n}\right)\left(y_{m+1} y_{m+2} y_{n}\right) \\
\quad \text { since }\left(F^{2}\right)^{3}=0 & \\
= & \left(y_{1} y_{2} \cdots y_{m}\right)\left(y_{m+1} y_{m+2}\right) \\
& +\left(y_{1} y_{2} \cdots y_{m}\right)\left(y_{m+1} y_{m+2}\right) y_{n} \\
& -\left(y_{1} y_{2} \cdots y_{m} y_{n} y_{n}\right)\left(y_{m+1} y_{m+2}\right)+\left(y_{1} y_{2} \cdots y_{m} y_{n}\right)\left(y_{m+1} y_{m+2}\right) y_{n} \\
\text { by the Jacobi identity } & \text { since }\left(F^{2}\right)^{2} F=0 . \\
= & \left(y_{1} y_{2} \cdots y_{m}\right)\left(y_{m+1} y_{m+2}\right) \\
& -\left(y_{1} y_{2} \cdots y_{m} y_{n} y_{n}\right)\left(y_{m+1} y_{m+2}\right)
\end{aligned}
$$

Hence $\left(y_{1} y_{2} \cdots y_{m} y_{n} y_{n}\right)\left(y_{m+1} y_{m+2}\right)$ is in the fully invariant ideal generated by $\left(y_{1} y_{2} \cdots y_{m}\right)\left(y_{m+1} y_{m+2}\right)$. Suppose that $n>m+2$ and substitute $y_{n+1}+y_{n+2}$ for $y_{n}$. We obtain

$$
\begin{aligned}
\left(y_{1} y_{2} \cdots y_{m} y_{n+1} y_{n+1}\right)\left(y_{m+1} y_{m+2}\right) & +\left(y_{1} y_{2} \cdots y_{m} y_{n+2} y_{n+2}\right)\left(y_{m+1} y_{m+2}\right) \\
& +\left(y_{1} y_{2} \cdots y_{m} y_{n+1} y_{n+2}\right)\left(y_{m+1} y_{m+2}\right) \\
& +\left(y_{1} y_{2} \cdots y_{m} y_{n+2} y_{n+1}\right)\left(y_{m+1} y_{m+2}\right) \\
= & \left(y_{1} y_{2} \cdots y_{m} y_{n+1} y_{n+1}\right)\left(y_{m+1} y_{m+2}\right) \\
& +\left(y_{1} y_{2} \cdots y_{m} y_{n+2} y_{n+2}\right)\left(y_{m+1} y_{m+2}\right) \\
& +2\left(y_{1} y_{2} \cdots y_{m} y_{n+1} y_{n+2}\right)\left(y_{m+1} y_{m+2}\right)
\end{aligned}
$$

by the Jacobi identity, since $\left(F^{2}\right)^{3}=0$.

Now the characteristic of $K$ is not 2, and so the fully invariant ideal of $F$ generated by $\left(y_{1} y_{2} \cdots y_{m}\right)\left(y_{m+1} y_{m+2}\right)$ contains $\left(y_{1} y_{2} \cdots y_{m} y_{n+1} y_{n+2}\right)\left(y_{m+1} y_{m+2}\right)$. By induction, if $r \equiv 0 \bmod 2$ the fully invariant ideal of $F$ generated by $\left(y_{1} y_{2} \cdots y_{m}\right)$ $\left(y_{m+1} y_{m+2}\right)$ contains $\left(y_{1} y_{2} \cdots y_{m} y_{n+1} \cdots y_{n+r}\right)\left(y_{m+1} y_{m+2}\right)$.

Now let 


$$
(i, j, k, l ; \boldsymbol{\alpha})=\left(y_{i}, y_{j} y_{1}^{\alpha_{1}} \cdots y_{m}^{\alpha_{m}}\right)\left(y_{k} y_{l}\right)
$$

and let $\sum_{n=1}^{\infty} \beta_{n}=r$. Then by the above remarks, provided $n>i, j, k, l, m$, the fully invariant ideal generated by $(i, j, k, l ; \boldsymbol{\alpha})$ contains

$$
\left(y_{i} y_{j} y_{1}^{\alpha_{1}} \cdots y_{m}^{\alpha_{m}} y_{n+1} y_{n+2} \cdots y_{n+r}\right)\left(y_{k} y_{1}\right)
$$

and so contains

$$
(i, j, k, l ; \boldsymbol{\alpha}+\boldsymbol{\beta})=\left(y_{i} y_{j} y_{1}^{\alpha_{1}} \cdots y_{m}^{\alpha_{m}} y_{1}^{\beta_{1}} \cdots y_{s}^{\beta_{s}}\right)\left(y_{k} y_{l}\right)
$$

where $s$ is chosen so that $\beta_{r}=0$ for $r>s$.

Corollary. If $\boldsymbol{\beta}=\left(\beta_{1}, \beta_{2}, \cdots\right) \in A$ and if $\sum_{n=1}^{\infty} \beta_{n} \equiv 0 \bmod 2$ then the fully invariant ideal generated by

contains

$$
g=\sum_{m=1}^{n} \lambda_{m}\left(i_{m}, j_{m}, k_{m}, l_{m} ; \alpha_{m}\right)
$$

$$
\sum_{m=1}^{n} \lambda_{m}\left(i_{m}, j_{m}, k_{m}, l_{m} ; \boldsymbol{\alpha}_{m}+\boldsymbol{\beta}\right)
$$

Proof. Apply the proof of Lemma 1 to $g$.

LEMMA 2. (a) If $\phi \in \Phi$ and if $s, t \in S, s<t$ then $s \phi^{*}<t \phi^{*}$, where $\phi^{*}$ is the endomorphism of $F$ given by $y_{r} \phi^{*}=y_{r \phi}$ for $r=1,2, \cdots$.

(b) If $(i, j, k, l ; \boldsymbol{\alpha})<(p, q, r, s ; \boldsymbol{\beta})$ then $(i, j, k, l ; \boldsymbol{\alpha}+\gamma)<(p, q, r, s ; \boldsymbol{\beta}+\gamma)$ for all $\gamma \in A$.

The proof of Lemma 2 is straightforward.

LEMMA 3. If $g, h \in\left(F^{2}\right)^{2}$ and if $w t g \preccurlyeq w t h$ then there is an element $g^{*}$ in the fully invariant ideal of $F$ generated by $g$ such that $\mathrm{wtg}^{*}=w t h$.

Proof. Let $w t g=(i, j, k, l ; \boldsymbol{\alpha}), w t h=(p, q, r, s ; \boldsymbol{\beta})$ and let $\phi \in \Phi$ satisfy

(1) $i \phi=p, j \phi=q, k \phi=r, l \phi=s$,

(2) $\alpha \phi \leqq \beta$,

(3) $\sum_{n=1}^{\infty} \alpha_{n} \equiv \sum_{n=1}^{\infty} \beta_{n} \bmod 2$.

Let $\phi^{*}$ be the endomorphism of $F$ determined by $y_{n} \phi^{*}=y_{n \phi}$ for $n=1,2, \cdots$. Then

$$
(i, j, k, l ; \boldsymbol{\alpha}) \phi^{*}=(p, q, r, s ; \boldsymbol{\alpha} \phi)
$$

and by Lemma 2 this is $w t\left(g \phi^{*}\right)$.

Since $\sum_{n=1}^{\infty} \alpha_{n} \equiv \sum_{n=1}^{\infty} \beta_{n} \bmod 2$, by the corollary to Lemma 1, and by Lemma 2 , the fully invariant ideal generated by $g \phi^{*}$ contains an element with weight 


$$
\begin{aligned}
(p, q, r, s ; \boldsymbol{\alpha} \phi & +(\boldsymbol{\beta}-\boldsymbol{\alpha} \phi)) \\
& =(p, q, r, s ; \boldsymbol{\beta}) \\
& =w t h .
\end{aligned}
$$

This completes the proof of Lemma 3.

Let $I$ be an ideal of $F$ contained in $\left(F^{2}\right)^{2}$. Since the set of weights of elements of $\left(F^{2}\right)^{2}$ is partially well ordered by $\preccurlyeq$ there is a finite subset $G \subseteq I$ with the property that for each $h \in I$ there is an element $g \in G$ such that $w t g \preccurlyeq w t h$.

Let $h \in I$ and let $g \in G, w t g \preccurlyeq w t h$. Then by Lemma 3 there is an element $g^{*}$ of the fully invariant ideal generated by $g$ such that $w t g^{*}=w t h$. But then for some $\lambda \in K w t\left(h+\lambda g^{*}\right)<w t h$. Since $\leqq$ is a well ordering on $S$ it follows, by induction on $w t h$, that $h$ is in the fully invariant ideal generated by $G$. This completes the proof of the proposition.

With minor modifications this proof gives the following result.

If $V$ is a variety of Lie algebras over a field $K$, if the characteristic of $K$ is not 2, and if $V$ satisfies the law

$$
\left(x_{1} x_{2}\right)\left(x_{3} x_{4}\right) x_{5} x_{5} \cdots x_{n}
$$

for some $n$, then $V$ has a finite basis for its laws.

\section{References}

[1] D. E. Cohen, 'On the laws of a metabelian variety', J. Algebra, 5 (1967), 267-273.

[2] Susan McKay, Some problems in group theory (Oxford D. Phil. thesis, 1970).

[3] M. R. Vaughan-Lee, Some varieties of Lie algebras (Oxford D. Phil. thesis, 1968)).

[4] M. R. Vaughan-Lee, 'Varieties of Lie algebras', Quart. J. Math. Oxford Ser. 21 (1970), 297308.

[5] M. R. Vaughan-Lee, 'Abelian-by-nilpotent varieties', Quart. J. Math. Oxford Ser. 21 (1970), 193-202.

University of Queensland

Australia

Present address

Christ Church, Oxford

England 